\title{
Image analysis techniques to characterise white clover root morphology
}

\author{
DEBBIE CARE $^{1}$, SHIRLEY NICHOLS ${ }^{1}$ and DEREK WOODFIELD ${ }^{2}$ \\ ${ }^{1}$ AgResearch, Ruakura Research Centre, Private Bag 3123, Hamilton \\ ${ }^{2}$ AgResearch, Grasslands Research Centre, Private Bag 11008, Palmerston North
}

\begin{abstract}
The use of low-ionic-strength hydroponic culture and image analysis techniques to discriminate and isolate morphologically distinct, genetically differentiated root types within white clover is described. Advantages of this method include the ability to view the genetic expression of the root systems without the modifying effects of growth in soil, to examine the growth and structure of roots over time, and to store the images for further examination. It is recognised that although the root systems grow in three dimensions, they are constrained to two dimensions by the flatbed scanner. However, the morphological parameters determined by image analysis would not be altered whether this analysis was measured in two or three dimensions.
\end{abstract}

Keywords: image analysis, root morphology, solution culture, Trifolium repens

\section{Introduction}

There is enormous variation in the form or architecture of root systems and some of this variation relates to their ecological adaptation (Fitter et al. 1991; Caradus 1990). For example, selections within white clover for increased tap-root diameter and increased proportion of root mass to total plant mass have exhibited improved growth and survival in drought-prone environments (Caradus \& Woodfield 1998).

It is difficult to measure differences in root morphology in soil because factors such as mechanical impedance and grazing by soil microfauna may modify the potential genetic expression of roots and their growth. In contrast, low-ionic-strength solution culture exposes the whole root to a homogeneous environment very similar in chemistry to the soil solution (Edmeades et al. 1985), and it is possible to assess the full genetic variation of entire root systems.

Combining low-ionic-strength hydroponic solution culture with image analysis techniques has enabled the magnitude of genetic variation for root morphological parameters within white clover lines, in the absence of modifications from edaphic factors, to be determined.
These techniques and their potential uses and benefits are outlined.

\section{Plant culture}

Scarified white clover seed was germinated on damp filter paper in covered petri dishes. Six days later healthy seedlings were suspended, with their roots passing through a hole in a rubber disc, into culture solution. Each unit was fixed in the lid of the culture tank by a plastic collar.

Fifteen plants were randomly placed in each of nine 451 tubs containing aerated, low-ionic-strength hydroponic solution (Table 1). Solution $\mathrm{pH}$ was maintained at $4.9( \pm 0.1)$. Nutrient levels were maintained by complete solution renewal, the timing of which depended on the growth rate of the plants.

Table 1 The final chemical concentration of the basal nutrient solution used in this method.

\begin{tabular}{cc}
\hline lons & Concentration $(\mu \mathrm{M})$ in the basal nutrient solution \\
\hline $\mathrm{Ca}^{2+}$ & 500 \\
$\mathrm{Mg}^{2+}$ & 100 \\
$\mathrm{Na}^{+}$ & 10 \\
$\mathrm{~K}^{+}$ & 300 \\
$\mathrm{NO}_{3}-\mathrm{N}$ & 450 \\
$\mathrm{NH}_{4}{ }^{+}-\mathrm{N}$ & 150 \\
$\mathrm{SO}_{4}^{2-}$ & 600 \\
$\mathrm{PO}_{4}{ }^{3-}$ & 4 \\
$\mathrm{Fe}^{3+}$ & 5 \\
$\mathrm{Mn}^{2+}$ & 6 \\
$\mathrm{Zn}^{2+}$ & 1 \\
$\mathrm{BO}_{4}{ }^{3-}$ & 1 \\
$\mathrm{Cu}^{2+}$ & 0.1 \\
\hline
\end{tabular}

Twice during the experiment, intact plants were removed from the pots, the roots and shoots scanned, and the longest root of each plant measured manually. Total plant fresh weight was recorded and the plants were then returned to the appropriate tub and position until final harvest.

After a final growth period, all plants were destructively harvested. The roots and shoots of each plant were removed from the pots, separated, blotted dry and weighed.

The roots and shoots were freeze dried and dry weights determined for each plant. 
Figure 1 Different stages of root image preparation using Video Pro 32 (Leading Edge Pty.). (a) the image of a clover root when scanned on a flatbed scanner (Microtek 600ZS) using a black back ground for contrast; (b) the image inverted using the negative menu function; (c) the threshold function used to give the root definition, is shown by a red overlay; (d) the image after the noise has been removed, and the image is ready for analysis.

(a)

colour

$53 \%$

(b)

colour

$53 \%$ 
(c)

colour

$53 \%$

(d)

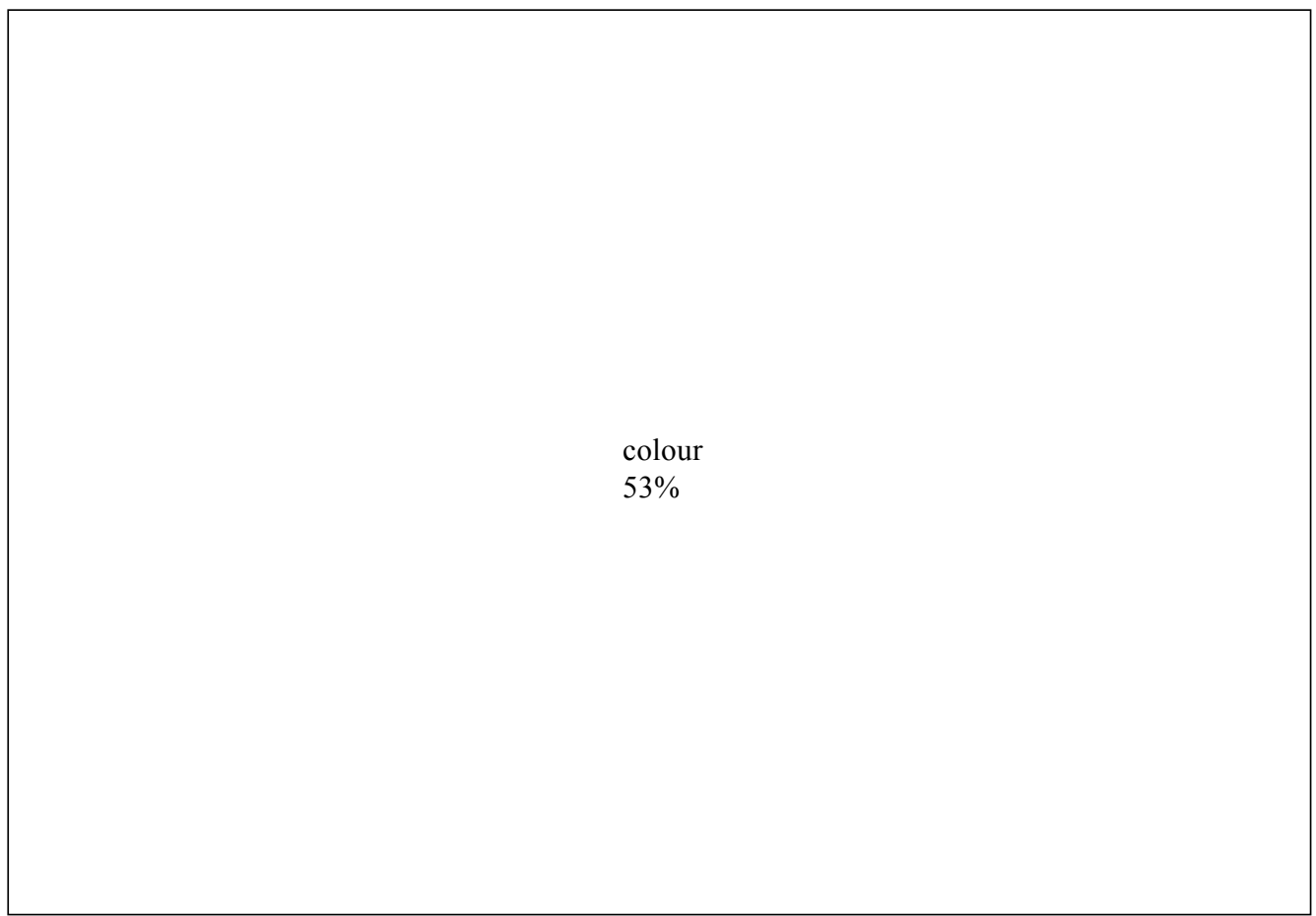




\section{Image analysis}

\section{Image capture}

The intact plants were placed on a flatbed scanner (Microtek 600ZS). Using the Twain Scan Module, Version 1.52, in Adobe Photoshop ${ }^{\mathrm{TM}}$ (Limited Edition $2.5)$, the root systems of each plant were then scanned as greyscale images. Image quality is critical to the accuracy of subsequent measurements, and preliminary experiments were done to define image quality determinants.

The image analysis package being used (Video Pro 32, Leading Edge Pty., - VP32) required that the size of the scanned images must be within $640 \times 442$ pixels, with a width divisible by four pixels. A $10 \mathrm{~mm}$ scale was scanned at the same settings, and calibrated in VP32 to produce a factor for subsequently converting pixel measurements to metric units.

\section{Analysis of images}

The image analysis program Video Pro 32 (VP32) was used to measure; the total root length, root perimeter (the outline of the root system), average root breadth and root scanned area of each plant at the two nondestructive harvests.

In VP32, maximum length is measured diagonally for a rectangular object, and breadth at $90^{\circ}$ to the length line. The perimeter and area of the root image were measured and used in the following calculations to obtain total length and average breadth of the roots:

$$
\begin{array}{ll}
\text { Length } & =\text { perimeter } / 2 \\
\text { Breadth } & =\text { area } /(\text { perimeter } / 2)
\end{array}
$$

Length and breadth calculations, along with measurements of perimeter, and root and shoot area, were performed on a whole-field basis by several command programs run within VP32. The accuracy of all measurements was validated using string, of known length, positioned to simulate a root system. Image quality was critical to the accuracy of the detected image and all subsequent measurements. The settings used in producing the images were determined through experimentation, to produce optimal appearance for the processing capabilities of VP32.

A command program automated the image analysis by selecting and applying a specified sequence of menu items and functions that the operator would normally perform manually. It also automates the creation and setup of data files. This program also sets variables to be measured (e.g., area and perimeter), assigns them to columns within the data file, incorporates desired calculations and initiates sequential image processing. Thus the user needs only to run the first program to fully process a batch of up to 99 images automatically.

\section{Root analysis}

Captured root images were first "inverted" using a negative function (Figure 1b). Pixels in greyscale images are assigned a level of grey from 0 to 254 , and features within the image can therefore be discriminated based on their grey values. In VP32 the range of values to be detected are defined by two thresholds and there are three detection modes available, in which the features discriminated are either darker than the lower threshold, brighter than the upper threshold, or lie between the two. By inverting the root images to a dark root on a light background (the negative function) the area of interest is darker than the lower threshold, or lightest level of grey selected. The appropriate level for this lower threshold was set by adjusting the histogram display command window in a range of the target images, and noting the change in detected features - shown on the image by a red overlay (Figure 1c). The histogram function is similar to a statistical histogram in principle - just as a statistical histogram illustrates the frequency at which a range of values occurs in a data set, the colour histogram command window shows the frequency of grey values in the displayed image. The selected thresholds are indicated by vertical lines, and the range of grey values that will be discriminated, according to the selected mode, are also overlain in red. The final threshold level selected was that which produced the most complete detection of the desired features, while minimising background noise, which must be excluded, before measuring the discriminated areas.

To remove small spots of noise a sizer function was included in the command program, which enables objects smaller or greater than specified values to be removed from the detected image. Sizing may be performed only on the variables being measured. Area and perimeter measurements were calculated for clusters of one to six pixels. For some groupings, it was possible for the pixels to be arranged in several ways, and thus have more than one perimeter or area measurement. For example, four pixels could take the form of a straight line, a block, an L shape, or one of several other possible arrangements. Each of these may alter the number of pixel sides that can be included in the perimeter measurement, and therefore more than one such measurement could be applied. A range of combinations was tested on a series of the target images, and compared to identify the best sizers. It was found that sizers based on the larger clusters of pixels removed more noise, but also began to remove sections of root. For the smaller pixel groupings there was little difference between two and four pixels, so it was decided to set sizers based on 
a feature the size of two adjacent pixels. A loss of this size from the root would not be as significant as that of a four-pixel section. The lower sizers were therefore $0.1716 \mathrm{~cm}$ for perimeter and $0.0016 \mathrm{~cm}^{2}$ for area. No upper sizers were set, as this would remove sections of the root system as well as larger areas of noise. Using only one or the other of the sizers did not exclude as many of the unwanted features, and this is why both were included. Each sizing is performed separately, and features do not have to meet both criteria to be excluded. The perimeter sizing was first, with any features smaller than the sizer being marked in blue and then removed completely. Any remaining features smaller than the area sizer are then marked and removed (Figure 1d).

To remove larger areas of noise, a measurement frame, which enables areas at the sides, top and bottom of the image to be excluded, was added to the command program. These larger areas of noise consistently occurred at the right and left hand edges of the images. The measurement frame was therefore sized and positioned to exclude a strip on each side of the image. The width of the screen covered by larger root systems, or the necessity of placing them sideways on the scanner, meant the position of the frame was sometimes adjusted to suit specific images. Water marks on some images created sizeable areas of noise that could not be removed by sizers or measurement frames. It was necessary to black out these areas using a graphics program before analysis in VP32.

\section{Potential uses and benefits}

Image analysis has become an integral part of our root research. When combined with low-ionic-strength hydroponics it has enabled us to isolate genetically expressed differences in root morphology. These morphological descriptions are important, and will be further extended to architectural analysis of these root systems. Architectural characteristics have been shown to have distinct functional attributes in roots (Fitter $e t$ al. 1991). The system as described above will be used to characterise and define differences in root morphology and architecture in drought-tolerant selections of white clover.

Carbon investment in root biomass may exceed 50\% of the total plant biomass. The image analysis technique is being used in tandem with measurements of carbon assimilation and flow, to define the carbon costs of white clover root systems. Detailed analyses of root systems, including root hairs, of two clover lines that varied in $\mathrm{P}$ response have been completed. The Presponsive genotype had a lower carbon investment per unit root surface area, and significantly higher shoot fresh weight, than the P non-responsive genotype (Care \& Caradus 1998)

Recently this system was used to complement measurements of nutrient uptake made by the Scanning Ion-selective Electrode Technique (SIET). This is a non-invasive technique which can be used to measure the uptake of mineral nutrients. The advantages of SIET include high resolution measurements for comparing different parts of a root system, e.g., root hairs, lateral roots and nodal roots to characterise their relative absorbing abilities. When used with the image analysis system it was possible to map spatial differences in proton uptake in nodulated and non-nodulated white clover roots.

Future work includes a modification of the culture system to examine the effect of pests on root morphology, and the effect of differences in root type on root predation. An important pest presently is the clover root weevil. Image analysis provides a good system for recording and quantifying the damage done by the root weevil and other root pests. It should be possible to calculate the loss of root surface area due to the clover root weevil.

\section{REFERENCES}

Caradus, J.R. 1990. The structure and function of white clover root systems. Advances in agronomy 43: 146.

Caradus, J.R.; Woodfield, D.R. 1998. Genetic control of adaptive root characteristics in white clover. Plant and soil 200: 63-69.

Care, D.A.; Caradus, J.R. 1998. Root hairs of white clover genotypes differing in phosphorus response. Phosphorus in plants colloquium, Penn State University, 28-30 May 1998.

Edmeades D.C., Wheeler D.M., and Clinton O.E. 1985. The chemical composition and ionic strength of soil solutions from New Zealand topsoils. Australian journal of soil research 23: 151-165.

Fitter A.H., Strickland T.R., Harvey M.L., and Wilson G.W. 1991. Architectural analysis of plant root systems 1. Architectural correlates of exploitation efficiency. New phytologist 118: 375-382. 
